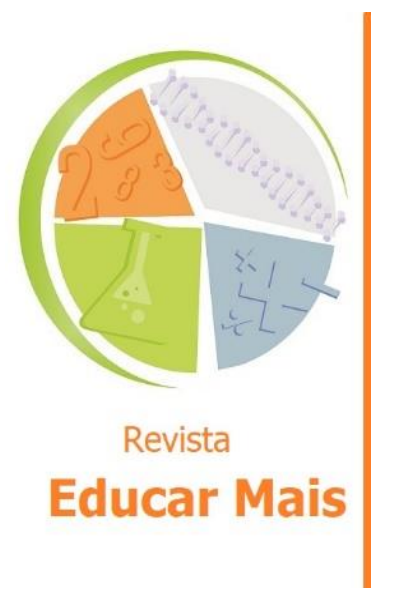

\section{O ensino da Sistematização da Assistência de Enfermagem sob uma perspectiva contemporânea da literatura}

\author{
Teaching the Systematization of Nursing Care under a contemporary \\ perspective of literature
}

\author{
Enseñar la Sistematización del cuidado de Enfermería bajo una \\ perspectiva contemporánea de la literatura
}

Luís Felipe Pissaia ${ }^{1}$; Arlete Eli Kunz da Costa²; Eniz Conceição Oliveira ${ }^{3}$

\begin{abstract}
RESUMO
Esse estudo possui o objetivo de analisar na literatura científica disponível como ocorre o ensino da Sistematização da Assistência de Enfermagem aos estudantes de enfermagem. Trata-se de uma revisão integrativa de literatura, realizada nos portais da Scientific Eletronic Library Online e no banco de teses e dissertações da CAPES. Foram analisados e discutidos treze artigos científicos, dissertações e tese selecionados, sendo utilizadas aproximações com a Análise de Conteúdo. Os resultados indicaram a importância de experiências no uso de tecnologias para o ensino da Sistematização da Assistência de Enfermagem, indicando o desenvolvimento de Objetos Virtuais de Aprendizagem. Evidenciou-se também a necessidade de introduzir o conteúdo nos currículos dos cursos técnicos em enfermagem, e por fim observou-se a aplicabilidade da Sistematização da Assistência de Enfermagem por meio da consulta realizada pelo enfermeiro. Sendo assim, este estudo possibilita analisar o panorama atual do ensino da Sistematização da Assistência de Enfermagem, tendo como enfoque a qualificação do ensino e da formação profissional.
\end{abstract}

Palavras-chave: Ensino em Enfermagem; Enfermagem; Processos de Trabalho; Ensino e Aprendizagem.

\begin{abstract}
This study aims to analyze in the available scientific literature how the teaching of Nursing Care Systematization occurs to nursing students. It is an integrative literature review, carried out on the portals of the Scientific Eletronic Library Online in Health Sciences and on the CAPES thesis and dissertation database. Thirteen scientific articles, dissertations and selected theses were analyzed and discussed, using approaches with Content Analysis. The results indicated the importance of experiences in the use of technologies for teaching Nursing Care Systematization, indicating the development of Virtual Learning Objects. The need to introduce the content in the curricula of technical courses in nursing was also evidenced, and finally, the applicability of the Systematization of Nursing Care was observed through the consultation performed by the nurse. Thus, this study makes it possible to analyze the current teaching panorama of the Nursing Care Systematization, focusing on the qualification of teaching and professional training.
\end{abstract}

Keywords: Nursing teaching; Nursing; Work Processes; Teaching and learning.

${ }^{1}$ Graduado em Enfermagem, Mestre em Ensino e Doutorando em Ensino pela Universidade do Vale do Taquari (Univates), Lajeado, RS - Brasil. E-mail: Ipissaia@universo.univates.br

${ }^{2}$ Doutora em Ambiente e Desenvolvimento e Professora da Universidade do Vale do Taquari (Univates), Lajeado, RS - Brasil. E-mail: arlete.costa@univates.br

${ }^{3}$ Pós-Doutora em Educação e Psicologia, Doutora em Química e Professora da Universidade do Vale do Taquari (Univates), Lajeado, RS - Brasil. E-mail: eniz@univates.br 


\section{RESUMEN}

Este estudio tiene como objetivo analizar en la literatura científica disponible cómo se da la enseñanza de la Sistematización del Cuidado de Enfermería a los estudiantes de enfermería. Se trata de una revisión integradora de la literatura, realizada en los portales de la Scientific Eletronic Library Online en la base de datos de tesis y disertaciones CAPES. Se analizaron y discutieron trece artículos científicos, disertaciones y tesis seleccionadas, utilizando enfoques con Análisis de Contenido. Los resultados indicaron la importancia de las experiencias en el uso de tecnologías para la enseñanza de la Sistematización del Cuidado de Enfermería, indicando el desarrollo de Objetos Virtuales de Aprendizaje. También se evidenció la necesidad de introducir los contenidos en los planes de estudio de los cursos técnicos en enfermería, y finalmente, se observó la aplicabilidad de la Sistematización de la Atención de Enfermería a través de la consulta realizada por la enfermera. Así, este estudio permite analizar el panorama docente actual de la Sistematización de la Atención de Enfermería, centrándose en la calificación de la docencia y la formación profesional.

Palabras clave: Enseñanza de enfermería; Enfermería; Procesos de trabajo; Enseñando y aprendiendo

\section{INTRODUÇÃO}

A enfermagem contemporânea é uma construção histórica que remonta a Idade Média e que perpassa as revoluções que a humanidade vivenciou ao longo dos séculos, para Moniz et al. (2020) o perfil de formação do enfermeiro está em constante reconstrução principalmente com a presença das tecnologias digitais. Para Schoeller (2020) a enfermagem é uma profissão em constante evolução, as melhorias na sua formação estão diretamente ligadas às necessidades do mercado de trabalho formando profissionais capacitados para a atuação nos diferentes cenários.

A capacitação profissional que o mercado de trabalho solicita atualmente vai ao encontro com os avanços tecnológicos de que toda a ampla área da saúde está se beneficiando principalmente com a presença das tecnologias digitais, uso de softwares e processos gerenciais conforme afirmam Backes et al. (2020). Os avanços tecnológicos são responsáveis pela disponibilidade de recursos eficazes para prolongar a expectativa de vida da população, por esse motivo Santos et al. (2020) defendem a qualificação da formação na área da enfermagem.

A formação em enfermagem segue o mesmo limiar de atualização profissional, sendo que a construção do conhecimento inicia-se com o bacharelado e permanece sendo revisitado com as práticas de educação permanente e continuada, conforme evidenciam Oliveira, Gazzinelli e Oliveira (2020). Atualmente, a formação do enfermeiro segue os três pilares básicos de atuação, sendo a assistência, o ensino e a gestão, segundo Reis et al. (2020) esses pontos se entrelaçam entre as diferentes disciplinas, estágios e práticas conferindo uma formação generalista e integral.

Dessa forma, para Nascimento (2020) a formação em enfermagem perpassa a necessidade de um ensino de qualidade que fomente a experimentação e a problematização dos estudantes, intuindo sobre a necessidade de refletir e estar presente na construção do conhecimento. $O$ ensino em enfermagem é construído por meio de conteúdos teóricos e práticos e segundo Oliveira, Gazzinelli e Oliveira (2020) a presença desses dois valores torna-se essencial para que o estudante vivencie o futuro profissional que o espera após o espaço acadêmico.

A estrutura curricular dos cursos de graduação em enfermagem evidencia o avanço científico e autonomia da profissão conforme comenta Duarte et al. (2020) incitando a identificação de processos que valorizam a atuação na área e demonstrem a qualificação presente nos serviços realizados com a população. Um dos meios de qualificar as práticas desenvolvidas pela área da enfermagem é a 
realização da Sistematização da Assistência de Enfermagem (SAE), que conforme Santos et al. (2020) trata-se de um processo de gestão eficaz do cuidado realizado pelos profissionais.

A SAE é um processo de gestão aplicado a todos os serviços de assistência à saúde realizados por profissionais da enfermagem segundo Souza et al. (2020) trata-se de uma metodologia de trabalho, cujo objetivo é reunir sistematicamente todas as informações necessárias ao plano de cuidados. 0 plano de cuidados é pensado sob diferentes aspectos e conforme Silva et al. (2020) é estruturado tendo como base o histórico de saúde e doença do indivíduo, seguido pelo estado de saúde atual, diagnósticos e as indicações de intervenções a serem realizadas para o cenário.

Assim, a realização da SAE pressupõe a aplicação do Processo de Enfermagem (PE), cujo objetivo é oferecer um passo a passo para a coleta de informações e realização do plano de cuidados conforme indicado por Santos et al. (2020). Segundo Tannure e Pinheiro (2014) o PE é estruturado em cinco etapas inter-relacionadas sendo: Histórico; Diagnósticos de Enfermagem; Prescrições de Enfermagem; Intervenções de Enfermagem e Avaliação.

Para Silva Consoline, Santos Lemos e Gomes (2020) a realização de toda a etapa do PE e sua reflexão sistemática infere sobre a aplicabilidade da $S A E$, sendo que o modelo de gestão baseia-se na reunião de informações e sua posterior análise e utilização em benefício dos processos de trabalho. Contudo, para que a SAE seja aplicada com eficácia os profissionais da enfermagem necessitam que a metodologia esteja presente durante a formação inicial e conforme indica Espírito Santo et al. (2020) a experimentação é a base para a compreensão integral e posterior realização.

Dessa forma, o ensino da SAE torna-se essencial para o desenvolvimento profissional da área da enfermagem, segundo Santos et al. (2020) é o método básico para que ocorra uma prática assistencial e de gestão efetiva nos serviços de saúde. Conforme Santana (2020) a SAE qualifica a assistência e oferece os subsídios científicos para que o plano de cuidados contemple as necessidades do indivíduo e da população sob o cuidado do enfermeiro.

Sendo assim, o objetivo desse estudo é analisar na literatura científica disponível como ocorre o ensino da SAE aos estudantes de enfermagem.

\section{METODOLOGIA}

O presente estudo estrutura-se como uma revisão integrativa de literatura, seguindo as aproximações com a metodologia de síntese de conhecimento e a incorporação da aplicabilidade de resultados proposta por Souza, Silva e Carvalho (2010). A revisão integrativa proposta pelos autores indica a presença de seis fases, sendo elas: a primeira elaboração da pergunta norteadora, a segunda a busca ou amostragem na literatura, a terceira a coleta de dados, a quarta a análise crítica dos estudos incluídos, a quinta a discussão dos resultados e a sexta a apresentação da revisão integrativa.

A pesquisa descrita nesse estudo ocorreu durante o primeiro semestre de 2020, iniciando-se as buscas de publicações científicas, bem como as suas respectivas leituras. Para a realização deste estudo definiu-se a seguinte questão de pesquisa: Como ocorre o ensino da Sistematização da Assistência de Enfermagem? 
Para a realização da pesquisa definiu-se como base de dados o portal da Scientific Eletronic Library Online (SciELO e o banco de teses e dissertações da CAPES. Os descritores utilizados foram "Sistematização da Assistência de Enfermagem" and "Ensino" and "Enfermagem".

Os critérios de inclusão para a busca foi todos os artigos científicos, dissertações ou teses que abordem o tema central da pesquisa; disponibilidade do material na íntegra online e gratuitamente; idiomas português, inglês e espanhol e publicado nos últimos cinco anos. E definiram-se como critério de exclusão, aqueles trabalhos que não se relacionem ao tema de pesquisa; aqueles publicados como trabalhos em eventos científicos, monografias ou livros. Observaram-se também casos específicos de duplicidade, sendo contabilizado somente um deles.

Em posse dos achados da pesquisa os artigos foram analisados e discutidos com aproximações na Análise de Conteúdo proposta por Bardin (2016). Sendo que para essa análise, os dados foram compilados e inseridos em um quadro sinóptico, contendo as informações de autor(es), título do artigo, periódico, ano, delineamento da pesquisa e procedência do estudo. As discussões apresentam as categorias emergentes da leitura flutuante dos artigos. Neste estudo observaram-se os aspectos éticos, respeitando os preceitos de autoria, bem como as citações de autores e/ou produções relacionadas à pesquisa.

\section{RESULTADOS}

Nesta seção são apresentados os resultados da pesquisa bibliográfica realizada descritos no Quadro 1 , sendo identificados por números ordinais romanos, indicando os seus autores, título, periódico em que o estudo foi publicado e o ano da publicação. No mesmo quadro é apresentado ainda o delineamento da pesquisa, que indica qual o tipo de estudo realizado e por fim a procedência do mesmo.

Quadro 01. Apresentação dos resultados da pesquisa bibliográfica.

\begin{tabular}{|c|c|c|c|c|c|c|}
\hline No & Autor(es) & Título do artigo & Periódico & Ano & $\begin{array}{c}\text { Delineamento } \\
\text { da pesquisa }\end{array}$ & $\begin{array}{l}\text { Procedência } \\
\text { do estudo }\end{array}$ \\
\hline I & $\begin{array}{c}\text { SALVADOR, P. T. } \\
\text { C. O.; RODRIGUES, } \\
\text { C. C. F. M.; } \\
\text { JÚNIOR, M. A. F.; } \\
\text { FERNANDES, M. I. } \\
\text { D.; MARTINS, J. C. } \\
\text { A.; SANTOS, V. E. } \\
\text { P. }\end{array}$ & $\begin{array}{c}\text { Construção de } \\
\text { hipermídia para } \\
\text { apoio ao ensino } \\
\text { da } \\
\text { sistematização } \\
\text { da assistência de } \\
\text { enfermagem }\end{array}$ & $\begin{array}{c}\text { Revista } \\
\text { Gaúcha de } \\
\text { Enfermagem }\end{array}$ & 2019 & $\begin{array}{c}\text { Desenvolviment } \\
\text { o de tecnologia } \\
\text { educacional }\end{array}$ & $\begin{array}{l}\text { Rio Grande do } \\
\text { Norte, Brasil }\end{array}$ \\
\hline II & $\begin{array}{l}\text { SILVA, S. O.; } \\
\text { MACHADO, L. M.; } \\
\text { SCHIMITH, M. D.; } \\
\text { SILVA, L. M. C. S.; } \\
\text { SILVEIRA, V. N.; } \\
\text { BASTOS, A. C. }\end{array}$ & $\begin{array}{l}\text { Consulta de } \\
\text { enfermagem às } \\
\text { pessoas com } \\
\text { Diabetes } \\
\text { Mellitus: } \\
\text { experiência com } \\
\text { metodologia } \\
\text { ativa }\end{array}$ & $\begin{array}{c}\text { Revista } \\
\text { Brasileira de } \\
\text { Enfermagem }\end{array}$ & 2018 & $\begin{array}{l}\text { Relato de } \\
\text { experiência }\end{array}$ & $\begin{array}{l}\text { Rio Grande do } \\
\text { Sul, Brasil }\end{array}$ \\
\hline
\end{tabular}




\begin{tabular}{|c|c|c|c|c|c|c|}
\hline No & Autor(es) & Título do artigo & Periódico & Ano & $\begin{array}{l}\text { Delineamento } \\
\text { da pesquisa }\end{array}$ & $\begin{array}{l}\text { Procedência } \\
\text { do estudo }\end{array}$ \\
\hline III & $\begin{array}{c}\text { SALVADOR, P. T. } \\
\text { C. O.; MARIZ, C. } \\
\text { M. S.; VIITOR, A. } \\
\text { F.; JÚNIOR, M. A. } \\
\text { F.; FERNANDES, M. } \\
\text { I. D.; MARTINS, J. } \\
\text { C. A.; SANTOS, V. } \\
\text { E. P. }\end{array}$ & $\begin{array}{c}\text { Validação de } \\
\text { objeto virtual de } \\
\text { aprendizagem } \\
\text { para apoio ao } \\
\text { ensino da } \\
\text { sistematização } \\
\text { da assistência de } \\
\text { enfermagem }\end{array}$ & $\begin{array}{c}\text { Revista } \\
\text { Brasileira de } \\
\text { Enfermagem }\end{array}$ & 2018 & $\begin{array}{c}\text { Estudo } \\
\text { quantitativo }\end{array}$ & $\begin{array}{l}\text { Rio Grande do } \\
\text { Norte, Brasil }\end{array}$ \\
\hline IV & $\begin{array}{c}\text { OLIVEIRA } \\
\text { SALVADOR, P. T. } \\
\text { C.; RODRIGUES, C. } \\
\text { C. F. M.; } \\
\text { BEZERRIL, M. S.; } \\
\text { FERREIRA, L. L.; } \\
\text { CHIAVONE, F. B. } \\
\text { T.; VIRGÍLIO, L. } \\
\text { A.; SANTOS, V. E. } \\
\text { P. }\end{array}$ & $\begin{array}{l}\text { Percepções de } \\
\text { profissionais de } \\
\text { enfermagem } \\
\text { acerca da } \\
\text { integração do } \\
\text { técnico de } \\
\text { enfermagem na } \\
\text { sistematização } \\
\text { da assistência }\end{array}$ & $\begin{array}{c}\text { Escola Anna } \\
\text { Nery Revista } \\
\text { de } \\
\text { Enfermagem }\end{array}$ & 2017 & $\begin{array}{c}\text { Estudo } \\
\text { descritivo, com } \\
\text { abordagem } \\
\text { qualitativa }\end{array}$ & $\begin{array}{l}\text { Região } \\
\text { Nordeste, } \\
\text { Brasil }\end{array}$ \\
\hline V & $\begin{array}{l}\text { BOAVENTURA, A. } \\
\text { P.; SANTOS, P. A.; } \\
\text { DURAN, E. C. M. }\end{array}$ & $\begin{array}{l}\text { Conocimiento } \\
\text { teórico-práctico } \\
\text { del enfermero } \\
\text { del Proceso de } \\
\text { Enfermería y } \\
\text { Sistematización } \\
\text { de Enfermería }\end{array}$ & $\begin{array}{l}\text { Enfermería } \\
\text { Global }\end{array}$ & 2017 & $\begin{array}{c}\text { Estudo } \\
\text { descritivo e } \\
\text { exploratório, } \\
\text { com abordagem } \\
\text { qualitativa }\end{array}$ & $\begin{array}{l}\text { São Paulo, } \\
\text { Brasil }\end{array}$ \\
\hline VI & $\begin{array}{c}\text { SALVADOR, P. T. } \\
\text { C. O;; VÍTOR, A. } \\
\text { F.; JÚNIOR, M. A. } \\
\text { F.; FERNANDES, M. } \\
\text { I. D.; SANTOS, V. } \\
\text { E. P. }\end{array}$ & $\begin{array}{c}\text { Ensinar } \\
\text { sistematização } \\
\text { da assistência de } \\
\text { enfermagem em } \\
\text { nível técnico: } \\
\text { percepção de } \\
\text { docentes }\end{array}$ & $\begin{array}{c}\text { Acta Paulista } \\
\text { de } \\
\text { Enfermagem }\end{array}$ & 2016 & $\begin{array}{c}\text { Estudo } \\
\text { descritivo, com } \\
\text { abordagem } \\
\text { mista }\end{array}$ & $\begin{array}{l}\text { Região } \\
\text { Nordeste, } \\
\text { Brasil }\end{array}$ \\
\hline VII & $\begin{array}{l}\text { MAZZO, M. H. S. } \\
\text { N.; BRITO, R. S. }\end{array}$ & $\begin{array}{l}\text { Instrumento para } \\
\text { consulta de } \\
\text { enfermagem à } \\
\text { puérpera na } \\
\text { atenção básica }\end{array}$ & $\begin{array}{c}\text { Revista } \\
\text { Brasileira de } \\
\text { Enfermagem }\end{array}$ & 2016 & $\begin{array}{c}\text { Pesquisa } \\
\text { metodológica }\end{array}$ & $\begin{array}{l}\text { Rio Grande do } \\
\text { Norte, Brasil }\end{array}$ \\
\hline VIII & $\begin{array}{l}\text { SALVADOR, P. T. } \\
\text { C. O.; SANTOS, V. } \\
\text { E. P.; BARROS, A. } \\
\text { G.; ALVES, K. Y. } \\
\text { A.; LIMA, K. Y. N. }\end{array}$ & $\begin{array}{c}\text { Ensino da } \\
\text { sistematização } \\
\text { da assistência de } \\
\text { enfermagem aos } \\
\text { técnicos de } \\
\text { enfermagem }\end{array}$ & $\begin{array}{c}\text { Escola Anna } \\
\text { Nery Revista } \\
\text { de } \\
\text { Enfermagem }\end{array}$ & 2015 & $\begin{array}{l}\text { Investigação } \\
\text { fenomenológica }\end{array}$ & $\begin{array}{l}\text { Rio Grande do } \\
\text { Norte, Brasil }\end{array}$ \\
\hline IX & $\begin{array}{l}\text { SILVA, J. P.; } \\
\text { GARANHANI, M. L.; } \\
\text { PERES, A. M. }\end{array}$ & $\begin{array}{c}\text { Sistematização } \\
\text { da Assistência de } \\
\text { Enfermagem na } \\
\text { graduação: um } \\
\text { olhar sob o } \\
\text { Pensamento } \\
\text { Complexo }\end{array}$ & $\begin{array}{c}\text { Revista } \\
\text { Latino- } \\
\text { Americana } \\
\text { de } \\
\text { Enfermagem }\end{array}$ & 2015 & $\begin{array}{l}\text { Estudo } \\
\text { qualitativo }\end{array}$ & Brasil \\
\hline
\end{tabular}




\begin{tabular}{|c|c|c|c|c|c|c|}
\hline No & Autor(es) & Título do artigo & Periódico & Ano & $\begin{array}{l}\text { Delineamento } \\
\text { da pesquisa }\end{array}$ & $\begin{array}{l}\text { Procedência } \\
\text { do estudo }\end{array}$ \\
\hline$x$ & MOURA, T. B. & $\begin{array}{c}\text { Criação de } \\
\text { software para } \\
\text { sistematização } \\
\text { da assistência de } \\
\text { enfermagem em } \\
\text { um hospital geral } \\
\text { de um município } \\
\text { baiano }\end{array}$ & $\begin{array}{c}\text { Dissertação } \\
- \\
\text { Universidade } \\
\text { Estadual de } \\
\text { Feira de } \\
\text { Santana }\end{array}$ & 2015 & $\begin{array}{l}\text { Pesquisa de } \\
\text { intervenção }\end{array}$ & $\begin{array}{l}\text { Estado da } \\
\text { Bahia, Brasil. }\end{array}$ \\
\hline XI & LOPES, R. O. P. & $\begin{array}{c}\text { Tecnologia } \\
\text { Educacional para } \\
\text { o Ensino dos } \\
\text { Diagnósticos de } \\
\text { Enfermagem } \\
\text { Sustentados } \\
\text { pelas Teorias de } \\
\text { Enfermagem em } \\
\text { Casos Clínicos }\end{array}$ & $\begin{array}{c}\text { Dissertação } \\
- \\
\text { Universidade } \\
\text { Federal do } \\
\text { Rio de } \\
\text { Janeiro }\end{array}$ & 2016 & $\begin{array}{l}\text { Desenvolviment } \\
\text { o de tecnologia } \\
\text { educacional }\end{array}$ & Brasil \\
\hline XII & $\begin{array}{l}\text { RODRIGUES, I. D. } \\
\text { C. V. }\end{array}$ & $\begin{array}{l}\text { Simulação } \\
\text { realística no } \\
\text { processo de } \\
\text { ensino- } \\
\text { aprendizagem do } \\
\text { raciocínio } \\
\text { diagnóstico de } \\
\text { enfermagem }\end{array}$ & $\begin{array}{c}\text { Tese - } \\
\text { Universidade } \\
\text { Federal do } \\
\text { Rio Grande } \\
\text { do Norte }\end{array}$ & 2017 & $\begin{array}{c}\text { Estudo } \\
\text { experimental, } \\
\text { do tipo ensaio } \\
\text { clínico } \\
\text { controlado e } \\
\text { randomizado }\end{array}$ & $\begin{array}{l}\text { Natal, Rio } \\
\text { Grande do } \\
\text { Norte, Brasil }\end{array}$ \\
\hline XIII & MACEDO, V. L. & $\begin{array}{l}\text { Validação de } \\
\text { conteúdo das } \\
\text { intervenções de } \\
\text { enfermagem } \\
\text { "ensino: pré- } \\
\text { operatório" e } \\
\text { "ensino: } \\
\text { procedimento/tra } \\
\text { tamento" da } \\
\text { classificação das } \\
\text { intervenções de } \\
\text { enfermagem, } \\
\text { para } \\
\text { procedimento } \\
\text { percutâneo em } \\
\text { hemodinâmica }\end{array}$ & $\begin{array}{c}\text { Dissertação - } \\
\text { Universidade } \\
\text { Estadual } \\
\text { Paulista Júlio } \\
\text { de Mesquita } \\
\text { Filho } \\
\text { (BOTUCATU) }\end{array}$ & 2016 & $\begin{array}{l}\text { Estudo } \\
\text { descritivo }\end{array}$ & Brasil \\
\hline
\end{tabular}

Fonte: Autores (2020).

O recorte temporal utilizado foi dos últimos cinco anos, sendo considerados para avaliação todos os artigos científicos publicados entre os anos 2015 e 2020. Iniciou-se a pesquisa no portal da SciELO, tendo como resultado dez artigos, sendo um descartado por incompatibilidade com o tema, resultando em nove artigos analisados nesse estudo. Após, realizou-se uma busca no banco de teses e dissertações da CAPES, resultado em 88601 itens, sendo que aplicados os critérios de inclusão e exclusão, restaram quatro.

Quanto aos anos de publicação, identificaram-se dois artigos e uma dissertação publicados em 2015, dois artigos e duas dissertações em 2016, dois artigos e uma tese em 2017, dois artigos em 2018 e um em 2019. Quanto aos periódicos das publicações, identificaram-se três artigos na Revista 
Brasileira de Enfermagem, dois na Escola Anna Nery Revista de Enfermagem e um artigo em cada uma das demais revistas: Revista Gaúcha de Enfermagem, Enfermería Global, Acta Paulista de Enfermagem e Revista Latino-Americana de Enfermagem. Quanto ao delineamento da pesquisa quatro artigos realizaram pesquisas qualitativas e em sua procedência, a totalidade foi realizada no Brasil. Quanto a procedência das dissertações, uma é da Universidade Estadual de Feira de Santana, uma da Universidade Federal do Rio de Janeiro e um da Universidade Estadual Paulista Júlio de Mesquita Filho, contabilizando também uma tese da Universidade Federal do Rio Grande do Norte.

\section{DISCUSSÕES}

Nesta seção serão discutidos os resultados obtidos com a pesquisa bibliográfica apresentada na seção anterior. A primeira categoria é intitulada "Uso de tecnologias para o ensino da Sistematização da Assistência em Enfermagem" sendo um espaço destinado para a identificação do uso de tecnologias com a finalidade de aplicar a SAE. A segunda categoria é intitulada "Ensino da Sistematização da Assistência de Enfermagem para técnicos em enfermagem" perfazendo sobre a importância no ensino da SAE para os cursos técnicos em enfermagem e, por fim a terceira categoria denominada "A Sistematização da Assistência de Enfermagem e a aplicabilidade na área da enfermagem" pactuando sobre as diferentes possibilidades de implementação da SAE nos serviços de saúde.

\subsection{Uso de tecnologias para o ensino da Sistematização da Assistência de Enfermagem}

Nesta categoria são apresentados e discutidos os achados de número I, III e X. Dessa forma, o uso de tecnologias no ensino em saúde é uma realidade contemporânea e conforme Santos et al. (2020) pressupõe uma necessidade para que a formação profissional seja de qualidade e correspondente com a necessidade da população. A incorporação de tecnologias no ensino fortalece a interação entre teoria e prática, dessa forma a contextualização proposta para os estudantes é capaz de favorecer a aprendizagem, conforme indicam Backes et al. (2020).

Os resultados demonstram que a utilização de tecnologias é um processo existente na área da saúde, conforme evidenciado no artigo I de Salvador et al. (2019) que buscou descrever o processo de construção de hipermídia para apoiar o ensino da SAE. Os autores buscaram desenvolver uma tecnologia educacional composta por conteúdo interativo, o qual é orientado por um mapa conceitual que direciona o aprendizado sobre o tema, intuindo assim sobre o fazer da SAE.

Segundo Santos et al. (2020) a compreensão da SAE pressupõe o entendimento sobre a importância de sua realização para a qualificação do trabalho em enfermagem, sendo que a identificação das etapas do processo é a base para que o cuidado sistemático ocorra. Dessa forma, o estudo de Salvador et al. (2019) estrutura o mapa conceitual sob o prisma dos quatro aspectos estruturantes da $S A E$, sendo o primeiro composto pelo conceito, histórico e benefícios, seguido pelo segundo que infere nos aspectos éticos e legais, pelo terceiro que infere na operacionalização e por fim o PE que traz as etapas e a integração do técnico em enfermagem.

Dessa forma, a apresentação de um passo a passo para a realização da $\mathrm{SAE}$, facilita a compreensão dos profissionais, bem como os estudantes durante o processo de ensino e aprendizagem, conforme indica Nascimento (2020). Nesse cenário, o estudo III de Salvador et al. (2018) descreve o processo de validação de conteúdo de um Objeto Virtual de Aprendizagem para o apoio no ensino da SAE para estudantes de cursos técnicos em enfermagem. 
Os autores Salvador et al. (2018) realizaram uma pesquisa metodológica com abordagem quantitativa, utilizando o referencial da psicometria de Pasquali, realizado de março a julho de 2016, contando com duas etapas de Delphi. O estudo contou com a avaliação de oito juízes na primeira etapa de Delphi e de sete na segunda, nas quais as sete telas do Objeto Virtual de Aprendizagem foram avaliadas, sendo que o mesmo foi validado com um coeficiente de 0,96 para servir de apoio ao ensino da SAE.

Assim, verifica-se que o uso de tecnologias torna-se pertinente ao ensino da $S A E$, principalmente por oferecer a didática necessária para o estudante compreender sua validade ao trabalho, conforme preconizam Tannure e Pinheiro (2014). A dissertação, achado de número X realizada por Moura (2015) trata-se de uma pesquisa de intervenção, que buscou construir um software para aplicação da $\mathrm{SAE}$, utilizando da engenharia de software e aproximando a tecnologia da. A presença de tecnologias em sala de aula é inerente à contemporaneidade, sendo responsabilidade do docente estruturar uma metodologia de ensino que contemple a utilização dessas ferramentas e comportem a eficácia do ensino dos conteúdos, conforme indicam Duarte et al. (2020).

O uso de ferramentas tecnológicas favorece a realização da $S A E$, seja por meio de softwares ou a própria manipulação de informações de maneira sistematizada. A gestão contemporânea dos serviços de saúde busca promover a sustentabilidade do negócio e oferecer a segurança necessária ao indivíduo que recebe os cuidados da equipe de enfermagem, sendo imprescindível o apoio tecnológico nesse meio, sendo fomentado durante a fase acadêmica para sua posterior implementação.

\subsection{Ensino da Sistematização da Assistência de Enfermagem para técnicos em enfermagem}

Nesta categoria são apresentados e discutidos os achados de número IV, VI e VIII. O limiar de realização de SAE perpassa pelo próprio exercício profissional da enfermagem, sendo que conforme Santos et al. (2020) a sistematização das informações obtidas junto ao processo assistencial é de responsabilidade privativa do enfermeiro, seguindo a legislação vigente. No que tange a responsabilização do técnico em enfermagem, Tannure e Pinheiro (2014) inferem que a sua participação é imprescindível para a coleta de dados para o PE e manutenção do bem-estar do indivíduo sob os cuidados da equipe de enfermagem.

Por vezes, o ensino da SAE para técnicos em enfermagem é suprimido ou deficitário devido à gestão do processo estar centrada na figura do enfermeiro, mas segundo Tannure e Pinheiro (2014) a atuação adequada de toda a equipe de enfermagem é imprescindível para a qualidade no cuidado prestado para a população. Dessa forma, o estudo IV de Oliveira Salvador et al. (2017) objetivou descrever as percepções de docentes e de profissionais da assistência à saúde acerca da integração do técnico em enfermagem na SAE.

Oliveira Salvador et al. (2017) apresentam uma pesquisa descritiva com abordagem qualitativa, realizada em um hospital universitário na região Nordeste do Brasil, tendo como participantes sete docentes e 13 técnicos em enfermagem. O estudo identificou as diferentes potencialidades da integração do técnico em enfermagem durante a realização da $\mathrm{SAE}$, inferindo sobre algumas dificuldades e fomentando a necessidade de reforçar o ensino profissional.

O mesmo estudo identificou que há várias possibilidades de atuação do técnico em enfermagem na realização da SAE e as contribuições estão de acordo com a importância do profissional em auxiliar 
nessa metodologia de trabalho. Para Santos et al. (2020) o engajamento da equipe de enfermagem é a base necessária para que a metodologia de trabalho seja implementada com sucesso e fomente a melhoria nos processos de cuidado à saúde prestado pelos serviços de saúde.

A mesma temática é abordada no artigo VI, onde os autores Salvador et al. (2016) buscam descrever a percepção de docentes sobre o ensino da SAE no nível técnico em enfermagem. Foi realizada uma pesquisa descritiva, com abordagem mista, com um grupo focal com sete docentes de um curso técnico em enfermagem.

Para Salvador et al. (2016) os docentes conseguem perceber a importância de integrar o profissional com a realização da $\mathrm{SAE}$, intuindo com veemência a necessidade de incluir o tema na formação. Contudo, os resultados apontam para a dificuldade de os docentes encontrarem o espaço para o ensino da SAE nas disciplinas do curso técnico em enfermagem, desse modo, é necessário revisitar o currículo para inserir essa temática.

Para Backes et al. (2020) o trabalho em equipe pressupõe a existência de um ensino qualificado e condizente com as práticas multidisciplinares e interdisciplinares, construindo assim uma cultura de cooperação em prol da utilização de metodologias consistentes para o cuidado da população. Sob esse limiar, o estudo VIII de Salvador et al. (2015) objetivou compreender o ideal de técnicos em enfermagem sobre a SAE, utilizando-se do referencial teórico de Alfred Schutz.

Desse modo, Salvador et al. (2015) realizaram uma investigação fenomenológica, sendo a coleta de informações por meio de grupo focal com técnicos em enfermagem. Os resultados evidenciaram a importância do técnico em enfermagem para a realização da SAE, bem como a implementação dessa metodologia de trabalho para a qualificação da assistência, que segundo os autores há possibilidades de melhoria na atuação desse profissional no processo, mas a chave está no ensino básico durante a formação.

A atuação do técnico em enfermagem é imprescindível para a realização da SAE de maneira eficaz e resolutiva, sendo que em conjunto com o enfermeiro, o profissional articula o plano de cuidados por meio do PE. Contudo, para que isso ocorra, é necessário que o ensino nos cursos técnicos em enfermagem ocorra de forma íntegra e holística, explorando as potencialidades profissionais e fortalecendo o trabalho em equipe.

\subsection{A Sistematização da Assistência de Enfermagem e a aplicabilidade na área da enfermagem}

Nesta categoria são apresentados e discutidos os achados de número II, V, VII, IX, XI, XII e XIII. A realização da SAE é defendida pela legislação vigente e conforme descrevem Tannure e Pinheiro (2014) estrutura-se como um processo intrincado de gestão do trabalho em enfermagem, de modo que os serviços de saúde ofereçam qualidade no plano de cuidado oferecido para a população. Para Santos, Montezeli e Peres (2012) a metodologia de trabalho que compõe a SAE é uma tecnologia em sua essência, sendo capaz de estruturar as bases de um cuidado científico e utilizar-se de ferramentas tecnológicas para a sua realização.

Uma das aplicabilidades da SAE é por meio da utilização do PE durante a consulta de enfermagem. Esse tema evidenciado no estudo II de Silva et al. (2018) cujo objetivo é descrever a experiência de utilização de metodologia da problematização para o ensino da consulta de enfermagem para pessoas 
com Diabetes Mellitus em uma Unidade de Saúde da Família. Os autores identificaram que a metodologia da problematização favoreceu o processo de ensino e aprendizagem, induzindo a uma compreensão ampliada do serviço de saúde a sua interação com o usuário.

Dessa forma, a consulta de enfermagem é um eixo que direciona a utilização da SAE, potencializando o plano de cuidados que se estrutura no PE, segundo Santos et al. (2020) a eficácia de sua utilização depende de um ensino de qualidade. Ainda para Silva et al. (2018), a realização da SAE permite conhecer a população acompanhada pela equipe, favorecendo o cuidado de enfermagem e desenvolvendo a autonomia profissional.

Sob o eixo do ensino da SAE e sua utilização, o estudo V de Boaventura, Santos e Duran (2017) objetivou identificar o conhecimento teórico e prático de enfermeiros sobre a SAE e o PE em um hospital universitário público do estado de São Paulo, Brasil. Os autores realizaram um estudo descritivo e exploratório com abordagem quali-quantitativa, identificando que os enfermeiros da instituição utilizam a SAE no seu contexto de trabalho e a aplicabilidade da metodologia contribui para as boas práticas assistenciais.

O conhecimento teórico e prático sobre a SAE confere aos estudantes um meio de experimentação e vivências necessárias para a formação profissional, conforme Nascimento (2020) esse é um meio de demonstrar a validação da metodologia de trabalho. Ao encontro disso, o estudo VII de Mazzo e Brito (2016) construiu um instrumento de consulta de enfermagem para mulheres puérperas na atenção básica. Para os autores o instrumento construído favorece a aplicação da SAE e infere sobre a utilização e aprendizagem de um método científico de avaliação em enfermagem, tal instrumento deriva de inquietações permanentes sobre a utilização da metodologia.

A presença da SAE na assistência à população é uma das preocupações dos currículos vigentes, dessa forma o artigo IX de Silva, Garanhani e Peres (2015) objetivou analisar as percepções de acadêmicos de um curso de graduação em enfermagem sobre a formação no que tange a metodologia de trabalho. Para os autores, que realizaram uma pesquisa qualitativa com 32 estudantes, ficou evidente que a SAE e o PE é trabalhado de forma fragmentada nas disciplinas do curso, sendo que a compreensão da metodologia ocorreu nas aulas práticas realizadas, reforçando a necessidade desses vivenciais que inter-relacionem a teoria com a prática acadêmica.

Dentre os achados também se encontram as dissertações XI e XIII de Lopes (2016) e Macedo (2016), respectivamente. A dissertação XI buscou desenvolver uma tecnologia educacional que integre os domínios teórico e metodológico no ensino dos diagnósticos de enfermagem em casos clínicos, tendo as teorias de enfermagem por sustentação. Sob o mesmo limiar, o achado XIII trata da validação de conteúdo das intervenções de enfermagem "Ensino: Pré-operatório" (5610) e "Ensino: Procedimento/Tratamento" (5618) da Classificação das Intervenções de Enfermagem (NIC), para procedimento percutâneo em hemodinâmica. Da mesma forma, a tese de Rodrigues (2017), achado XII teve uma amostra de 24 estudantes e buscou avaliar a eficácia da simulação realística no processo de ensino e aprendizagem do raciocínio diagnóstico de enfermagem. Considera-se que as realizações desses estudos possibilitam a qualificação das práticas desenvolvidas por enfermeiros, atuando diretamente na aplicabilidade dos estudos e integrando o desenvolvimento constante da área, conforme indicam Backes et al. (2020).

A realização da SAE nos serviços de saúde busca a qualificação das práticas de gestão assistencial, favorecendo a construção de um plano de cuidados efetivo e resolutivo a partir dos problemas 
vivenciados pelo indivíduo. A qualificação no ensino da SAE fundamenta sua correta utilização e aplicação nos serviços de saúde, intuindo que a relação entre teoria e prática fortalece a aprendizagem dos estudantes.

\section{CONSIDERAÇÕES FINAIS}

Este estudo buscou analisar na literatura científica disponível como ocorre o ensino da SAE aos estudantes de enfermagem. Dessa forma, foram analisados e discutidos nove artigos selecionados nas buscas bibliográficas no portal da SciELO e o banco de teses e dissertações da CAPES.

Os resultados trouxeram iniciativas de utilização de tecnologias como apoio para o ensino da $\mathrm{SAE}$, estimulando a criação de Objetos Virtuais de Aprendizagem, que se constituem em modelos a serem seguidos para a aprendizagem e implementação nos serviços de saúde. Os modelos de ensino e aprendizagem baseados em ferramentas tecnológicas facilitam a compreensão dos estudantes e profissionais, propiciando o desenvolvimento do conhecimento sobre o processo da SAE.

Outra questão destacada nos estudos selecionados é o ensino da SAE para os cursos técnicos em enfermagem, intuindo e articulando a importância desses profissionais para a metodologia de trabalho, sobretudo na qualificação da assistência oferecida para a população. Contudo, observou-se que os docentes possuem dificuldade em inserir o conteúdo no currículo dos cursos técnicos em enfermagem, declarando ciência de sua necessidade para a formação profissional.

E por fim, a busca no portal da SciELO e o banco de teses e dissertações da CAPES incitaram sobre a importância de aplicar a SAE como metodologia de trabalho aos profissionais da enfermagem, indicando a realização da consulta de enfermagem norteada com o PE. Nesse sentido, o PE constituise como uma maneira de auxiliar a implementação da SAE nos serviços de saúde, possibilitando um passo a passo metodológico para a construção do plano de cuidados realizado pela equipe de enfermagem ou ainda, a multiprofissional.

Sendo assim, esse estudo possibilitou compreender o cenário do ensino da SAE para a área da enfermagem, sendo possível verificar as suas potencialidades, experiências positivas e oportunidades de melhoria nos processos vigentes, de ensino e assistência em saúde. Esses resultados, responderam ao objetivo da pesquisa e oferecem o estímulo necessário para a realização de estudos futuros que abarquem a temática do ensino da $\mathrm{SAE}$, com vistas à qualificação do ensino e da formação profissional.

\section{REFERÊNCIAS}

BACKES, Dirce Stein; TOSON, Marcelo Junior; BEN, Luiza Watanabe Dal; ERDMANN, Alacoque Lorenzini. Contribuições de Florence Nightingale como empreendedora social: da enfermagem moderna à contemporânea. Revista Brasileira de Enfermagem, v. 73, 2020.

BARDIN, Laurence. Análise de Conteúdo. Tradução: Luís Augusto Pinheiro. São Paulo: Edições 70, 2016.

BOAVENTURA, Ana Paula; SANTOS, Pedro Alves; DURAN, Erika Christiane Marocco. Conocimiento teórico-práctico del enfermero del Proceso de Enfermería y Sistematización de Enfermería. Enfermería Global, v. 16, n. 46, p. 182-216, 2017. 
DUARTE, Evelin Leite; VASCONCELOS, Kariny Gonzaga; OLIVEIRA, Walas de Paula; GOMES, Katia Regina Bruno. Sistematização da Assistência de Enfermagem e a segurança do paciente. Revista Científica da Faculdade de Educação e Meio Ambiente, v. 10(Especial), p. 113-118, 2020.

ESPÍRITO SANTO, Ilana Maria Brasil; MATOS, Johnata da Cruz; SILVA, Charles José; ALMEIDA, Regiane dos Passos; SANTOS, Jéssica Larissa Pereira; SILVA, Sheila Maciel; CAETANO, Elielson dos Reis; LIMA, Roniel Dias; NUNES, Karoline Soares; BARBOSA, Sérgio da Silva. Sistematização da Assistência de Enfermagem Perioperatória (SAEP): Reflexos da Aplicabilidade no Processo de Cuidar. Revista Eletrônica Acervo Saúde, v. 43, p. e2945-e2945, 2020.

LOPES, Rafael Oliveira Pitta. Tecnologia Educacional para o Ensino dos Diagnósticos de Enfermagem Sustentados pelas Teorias de Enfermagem em Casos Clínicos. Dissertação. Universidade Federal do Rio de Janeiro, 2016.

MACEDO, Vanessa Luciana. Validação de conteúdo das intervenções de enfermagem "ensino: préoperatório" e "ensino: procedimento/tratamento" da classificação das intervenções de enfermagem, para procedimento percutâneo em hemodinâmica. Dissertação. Universidade Estadual Paulista Júlio de Mesquita Filho - BOTUCATU, 2016.

MAZZO, Maria Helena Soares da Nóbrega; BRITO, Rosineide Santana. Instrumento para consulta de enfermagem à puérpera na atenção básica. Revista Brasileira de Enfermagem, v. 69, n. 2, p. 316-325, 2016.

MONIZ, Marcela de Abreu; DAHER, Donizete Vago; SABÓIA, Vera Maria; RIBEIRO, Crystiane Ribas Batista. Saúde ambiental: desafios e possibilidades para o cuidado emancipador pelo enfermeiro. Revista Brasileira de Enfermagem, v. 73, n. 3, 2020.

MOURA, Tayse Barbosa. Criação de software para sistematização da assistência de enfermagem em um hospital geral de um município baiano. Dissertação. Universidade Estadual de Feira de Santana, 2020.

NASCIMENTO, Evany Rosário. Desafios da assistência de enfermagem ao parto humanizado. Caderno de Graduação-Ciências Biológicas e da Saúde-UNIT-SERGIPE, v. 6, n. 1, p. 141, 2020.

OLIVEIRA SALVADOR, Pétala Tuani Candido; RODRIGUES, Cláudia Cristiane Filgueira Martins; BEZERRIL, Manacés dos Santos; FERREIRA, Larissa de Lima; CHIAVONE, Flávia Barreto Tavares; VIRGÍLIO, Lílian de Andrade; SANTOS, Viviane Euzébia Pereira. Percepções de profissionais de enfermagem acerca da integração do técnico de enfermagem na sistematização da assistência. Escola Anna Nery Revista de Enfermagem, v. 21, n. 2, p. 1-9, 2017.

OLIVEIRA, Vânia Aparecida da Costa; GAZZINELLI, Maria Flávia; OLIVEIRA, Patrícia Peres. Articulação teórico-prática em um currículo de um curso de Enfermagem. Escola Anna Nery, v. 24, n. 3, 2020.

REIS, Nathália da Silva Pimentel; DIAS, Camila da Silva; MONTEIRO, Valéria de Oliveira; BARRETO, Carla Tatiana Garcia; SANTOS, Raíla de Souza; NUNES, Alessandra Sant'anna. Inserção da enfermagem nas tecnologias diagnósticas: inovações em atenção secundária de saúde. Revista de Pesquisa: Cuidado é Fundamental Online, v. 12, p. 27-33, 2020.

RODRIGUES, Iellen Dantas Campos Verdes. Simulação realística no processo de ensino-aprendizagem do raciocínio diagnóstico de enfermagem. Tese. Universidade Federal do Rio Grande do Norte, 2017.

SALVADOR, Pétala Tuani Candido de Oliveira; MARIZ, Camila Maria dos Santos; VÍTOR, Allyne Fortes; JÚNIOR, Marcos Antônio Ferreira; FERNANDES, Maria Isabel Domingues; MARTINS, José Carlos 
Amado; SANTOS, Viviane Euzébia Pereira. Validação de objeto virtual de aprendizagem para apoio ao ensino da sistematização da assistência de enfermagem. Revista brasileira de enfermagem, v. 71, n. 1, p. 11-19, 2018.

SALVADOR, Pétala Tuani Candido de Oliveira; RODRIGUES, Cláudia Cristiane Filgueira Martins; JÚNIOR, Marcos Antônio Ferreira; FERNANDES, Maria Isabel Domingues; MARTINS, José Carlos Amado; SANTOS, Viviane Euzébia Pereira. Construção de hipermídia para apoio ao ensino da sistematização da assistência de enfermagem. Revista Gaúcha de Enfermagem, v. 40, 2019.

SALVADOR, Pétala Tuani Candido de Oliveira; SANTOS, Viviane Euzébia Pereira; BARROS, Adriana Gonçalves; ALVES, Kisna Yasmin Andrade; LIMA, Kálya Yasmine Nunes. Ensino da sistematização da assistência de enfermagem aos técnicos de enfermagem. Escola Anna Nery Revista de Enfermagem, v. 19, n. 4, p. 557-562, 2015.

SALVADOR, Pétala Tuani Candido de Oliveira; VÍTOR, Allyne Fortes; JÚNIOR, Marcos Antônio Ferreira; FERNANDES, Maria Isabel Domingues; SANTOS, Viviane Euzébia Pereira. Ensinar sistematização da assistência de enfermagem em nível técnico: percepção de docentes. Acta Paulista de Enfermagem, v. 29, n. 5, p. 525-533, 2016.

SANTANA, Rosimere Ferreira. Sistematização da Assistência de Enfermagem, uma invenção brasileira?. Revista de Enfermagem e Atenção à Saúde, v. 8, v. 2, 2020.

SANTOS, Fernanda Batista Oliveira; SILVA, Iago Leonardo Vieira; DUTRA, Bianca Santana; SANTANA, Júlio César Batista; CARREGAL, Fernanda Alves dos Santos; BARBOSA, Jaqueline Almeida Guimarães. Saberes, desafios e perspectivas sobre a sistematização da assistência de enfermagem. Revista Enfermagem Contemporânea, v. 9, n. 1, p. 41-49, 2020.

SANTOS, Fernanda de Oliveira Florentino; MONTEZELI, Juliana Helena; PERES, Aida Maris. Autonomia profissional e sistematização da assistência de enfermagem: percepção de enfermeiros. Revista Mineira de Enfermagem, v. 16, n. 2, p. 251-257, 2012.

SCHOELLER, Soraia Dornelles. Enfermeiro e cuidado: a distância entre a intenção e a prática. Enfermagem Brasil, v. 10, n. 5, p. 280-288, 2020.

SILVA CONSOLINE, Letícia; SANTOS LEMOS, Larissa; GOMES, Katia Regina. Inovação na enfermagem: uma assistência de qualidade. Revista Científica da Faculdade de Educação e Meio Ambiente, v. 10(Especial), p. 55-60, 2020.

SILVA, Amina Regina; FORTE, Elaine Cristina Novatzki; PADILHA, Maria Itayra; VIEIRA, Amanda Nicácio. Contribuições da Sistematização da Assistência de Enfermagem ao Paciente Renal Crônico: Revisão Integrativa. Revista de Pesquisa: Cuidado é Fundamental Online, v. 11, n. 3, p. 700706, 2020.

SILVA, Josilaine Porfírio; GARANHANI, Mara Lucia; PERES, Aida Maris. Sistematização da Assistência de Enfermagem na graduação: um olhar sob o Pensamento Complexo. Revista latino-americana de enfermagem, v. 23, n. 1, p. 59-66, 2015.

SILVA, Silvana de Oliveira; MACHADO, Letícia Martins; SCHIMITH, Maria Denise; SILVA, Laís Mara Caetano; SILVEIRA, Vanessa do Nascimento; BASTOS, Anderson Cecchin. Consulta de enfermagem às pessoas com Diabetes Mellitus: experiência com metodologia ativa. Revista brasileira de enfermagem, v. 71, n. 6, p. 3103-3108, 2018.

SOUZA, Guilherme Barbosa; SILVA SANTIAGO, Ana Xavier; SANTOS, Osmar Pereira; PEREIRA, Bruno Alves; SOUZA CAETANO, Sandra Rosa; SANTOS, Clarice Carvalho. Sistematização da assistência de 
enfermagem e processo de enfermagem: conhecimento de graduandos. Brazilian Journal of Health Review, v. 3, n. 1, p. 1250-1271, 2020.

SOUZA, Marcela Tavares; SILVA, Michelly Dias; CARVALHO, Rachel. Revisão integrativa: o que é e como fazer. Einstein. v. 8, (1 Pt 1), p. 102-6, 2010.

TANNURE, Meire Chucre; PINHEIRO, Ana Maria. SAE: Sistematização da Assistência de Enfermagem: Guia Prático. Rio de Janeiro, Guanabara Koogan, 2014.

Submissão: 02/02/2021

Aceito: 08/03/2021 